\title{
INEQUALITIES FOR BRACHISTOCHRONE
}

\author{
A. G. RAMM
}

Abstract. Let $A=(0,1)$ and $B=(b, 0)$ be the initial and final points, and $y=y(x)$ joins $A$ with $A B, y^{\prime \prime} \geqslant 0,0 \leqslant y(x) \leqslant y_{0}(x)$, where $y_{0}(x)$ is the straight line joining $A$ and $B$. Denote by $S$ the set of such $y(x)$. Let $P$ be the polygon consisting of two segments of straight lines: $A 0$ and $O B$, where $0=(0,0)$ is the origin. Up to a constant factor depending on the choice of units, the time needed for a particle to get from $A$ to $B$ along $y(x)$ in the gravitational field is $T(y)=\int_{0}^{b} \frac{\sqrt{1+y^{\prime}}}{\sqrt{1-y}} d x$. Let $T_{0}:=T\left(y_{0}\right)=2 \sqrt{1+b^{2}}, T_{P}:=T(P)=2+b$. It is conjectured that:

1) if $0<b<\frac{4}{3}$ then $T\left(y_{b r}\right) \leqslant T(y)<T_{P}, y \in S$,

2) if $\frac{4}{3} \leqslant b \leqslant \frac{\pi}{2}$ then $T\left(y_{b r}\right) \leqslant T(y) \leqslant T_{0}, y \in S$,

3) if $b>\frac{\pi}{2}$ then $T\left(P_{b r}\right)<T(y) \leqslant T_{0}, y \in S$

where $y_{b r}=y_{b r}(x) \in S$ is the classical brachistochrone curve. For $b>\frac{\pi}{2}$ this curve probably degenerates into $P_{b r}$, the brachistochrone curve which joins $A$ and $(\pi / 2,0)$ and the straight line joining $(\pi / 2,0)$ and $(b, 0)$.

Mathematics subject classification (1991): 49A36, 49A05.

Key words and phrases: Calculus of variations, inequalities, brachistochrone.

\section{REFERENCES}

[1] I. Gelfand, S. Fomin, Calculus of Variations, Prentice Hall, Englewood Cliffs, 1963.

[2] L. CESARI, Optimization theory and applications, Springer Verlag, Berlin, 1983. 